\title{
Description de l'adulte présumé de Bursotrema tetracotyloides Szidat, 1960, et d'une nouvelle espèce du genre Didelphodiplostomum Dubois, 1944, parasites de Didelpbis azarae Temminck, 1825 (Trematoda : Alariinae)
}

par G. DUBOIS

Grand-Rue 12, CH-2035 Corcelles.

\section{Résumé.}

L'auteur décrit deux Trématodes sud-américains (Diplostomidae: Alariinae), récoltés par la Dra Margarita O. de Núñez dans un Opossum, Didelphis azarae Temminck, 1825, aux environs de Buenos Aires. L'un de ceux-ci permet de définir le genre larvaire Bursotrema Szidat, 1960. L'autre représente une nouvelle espèce du genre Didelphodiplostomum Dubois, 1944.

Summary.

Description of the supposed adult of Bursotrema tetracotyloides Szidat, 1960, and of a new species of the genus Didelphodiplostomum Dubois, 1944, parasites from Didelphis azarae Temminck, 1825 (Trematoda : Alariinae).

The author describes two South American trematodes (Diplostomidae: Alariinae) collected by Dra Margarita O. de Núñez from a opossum, Didelphis azarae Temminck, 1825, in the vicinity of Buenos Aires. One of them permits to define the larval genus Bursotrema Szidat, 1960. The other one is a new species of the genus Didelphodiplostomum Dubois, 1944. 


\section{Introduction}

Deux matériels récoltés le 12 mai 1975, dans l'intestin d'un Opossum, Didelphis azarae Temminck, 1825, capturé à Castelar (prov. de Buenos Aires), nous ont été envoyés par la $D^{\text {ra }}$ Margarita Ostrowski de Núñez, de Boulogne, F.C.N.G.B. (Argentine). L'un contenait plusieurs exemplaires et une série de coupes histologiques d'un Alariiné que nous considérons comme la forme adulte de Bursotrema tetracotyloides Szidat, 1960 ; l'autre représente une espèce nouvelle appartenant au genre Didelphodiplostomum Dubois, 1944.

Nous remercions $\mathbf{M}^{\mathrm{m}}$ Ostrowski de Núñez de nous avoir confié l'étude de ces matériels et d'avoir attiré notre attention sur le travail de Lothar Szidat, relatif au genre Bursotrema.

\section{Diagnose du genre Bursotrema Szidat, 1960}

\section{(stade adulte)}

Diplostomidae: Alariinae à corps ascidiforme, à segment antérieur utriculaire, sans pseudo-ventouses, à ouverture oblique et dont la paroi interne s'épaissit à partir d'un bourrelet sphinctéroïde situé un peu en avant du niveau où débutent les vitellogènes, pour enserrer la base de l'organe tribocytique pistilliforme, canaliculé et entaillé ventro- transversalement, et, au-delà, latéro-ventralement, la masse du segment postérieur qui contient les glandes génitales. Bourse copulatrice très grande et très rétractile, à parois épaisses et à large ouverture dorsale, subterminale, — abritant un cône génital précédé d'un sinus ramifié, où débouche le ductus ejaculatorius, et qui se prolonge en direction axiale pour recevoir le métraterme (à 65 ou $70 \mu$ du pore mâle) et constituer avec lui le canal hermaphrodite. Ovaire dorso-latéral, contigu au premier testicule; second testicule plus développé, bilobé, coiffant une vésicule séminale volumineuse. Vitellogènes occupant la partie moyenne du corps et s'étendant ventralement jusqu'à la hauteur du testicule postérieur. Réservoir vitellin intertesticulaire. Parasites intestinaux de Marsupiaux (Didelphidae).

Espèce type: Bursotrema tetracotyloides Szidat, 1960.

Bursotrema tetracotyloides, Szidat, 1960 (fig. I).

Szidat (1960, pp. 84-87) avait décrit sous ce nom une métacercaire trouvée en grand nombre dans le rein de Leptodactylus ocellatus (L.). Ses préparations sont considérées comme perdues (lettre de la $\mathrm{D}^{\text {ra }} \mathrm{O}$. de Núñez, 16-XI-1975). Mais $\mathbf{M}^{\mathrm{me}}$ Ostrowski 
de Núñez a retrouvé 7 exemplaires de Bursotrema tetracotyloides dans le rein d'un jeune Leptodactylus ocellatus, capturé aux environs de Buenos Aires (lettre du 8-I-1976).

DiAGNOSE DE L'AdULTE (avec les caractères du genre). - Corps ascidiforme (1), spinescent jusqu'au tiers ou au quart postérieur, long de 0,95 $0,97 \mathrm{~mm}$ à l'état normal (de 0,88 $0,94 \mathrm{~mm}$ à l'état contracté), large de $0,27-0,30 \mathrm{~mm}(0,35-0,38 \mathrm{~mm})$ dans son premier tiers, de $0,43-0,50 \mathrm{~mm}$ $(0,47-0,53 \mathrm{~mm})$ au niveau du segment postérieur. Ventouse buccale $55-73 / 57-73 \mu$; pharynx très musculeux, 65-68/50-55 u; ventouse ventrale faible, 37-40/52-57 $\mu$, située assez en avant, non ou en partie recouverte par l'organe tribocytique qui mesure 250-260 $\mu$ de longueur, $120-130 \mu$ de largeur dans sa partie stigmatiforme, 68-75 $\mu$ à mi-longueur et $140-160 \mu$ à sa base. Esophage nul ; caeca s'allongeant jusqu'audevant de la bourse copulatrice. Ovaire dextre, 65-90/120-150 $\mu$; testicule antérieur 75-105/155-170 $\mu$, médian ou latéral; testicule postérieur mesurant 320-370 $\mu$ dans le sens iransversal, $110-140 \mu$ et 150 . $160 \mu$ dans le sens longitudinal (pour l'un et l'autre de ses lobes). Cône génital haut de $65 \mu$, large de $115 \mu$ à sa base, retiré au fond de l'atrium. Eufs 80-100/45-55 $\mu$, dont le nombre peut atteindre la dizaine (fig. 2).

HôTE: Didelphis azarae Temmick, 1825 .

Habitat : intestin.

Localité: Castelar, prov. Buenos Aires (Argentine).

Matériel: Museo Argentino de Ciencias Naturales « Bernardino Rivadavia », av. Angel Gallardo 470, Buenos Aires, coll. helm. $\mathrm{n}^{\circ}$ 274/1-5 (O. de Núñez), 274/6 (hototype et 2 para-

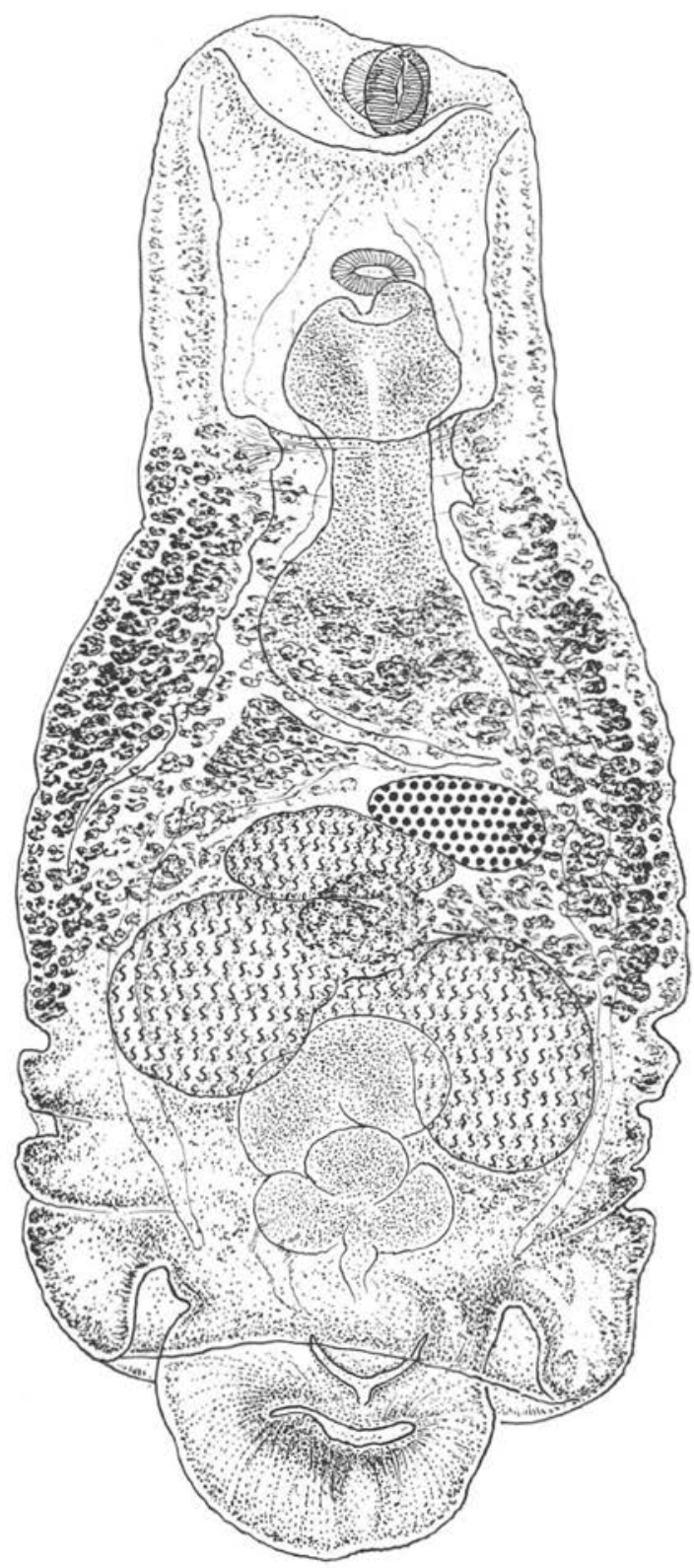

FIG. 1. - Bursotrema tetracotyloides Szidat, 1960, de Didelphis azarae Temm. Holotype. Longueur $0,95 \mathrm{~mm}$. Vue dorsale.

(1) Le nom spécifique, donné par Szidat à la métacercaire, ne caractérise pas la forme adulte. 


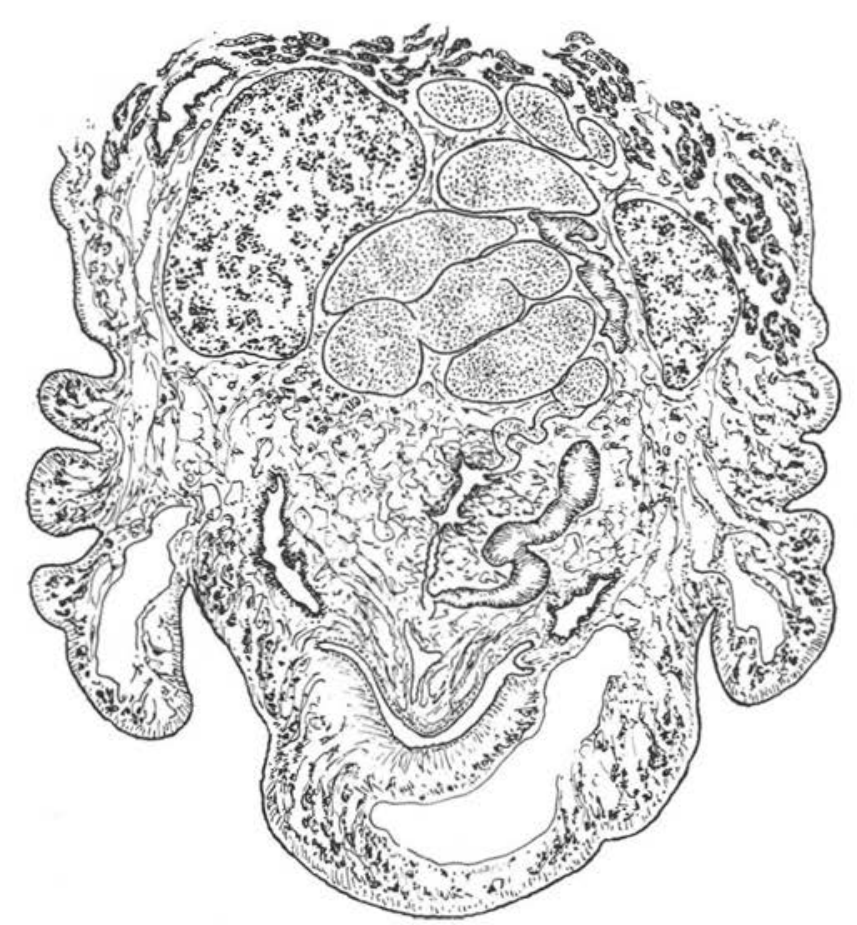

FIG. 2. - Bursotrema tetracotyloides Szidat, 1960. Paratype. Coupe composite d'orientation intermédiaire entre les plans horizontal et dorso-ventral.

types), 274/7 (3 paratypes), 274/8 (coupes histologiques); Université de Neuchâtel (G.D.), $\mathrm{n}^{\circ}$ W 18 et 19 (paratypes).

Discussion. - Ce parasite de l'Opossum est considéré présomptivement comme l'adulte de la métacercaire trouvée par Szidat (1960) dans le rein de Leptodactylus ocellatus (L.), en Argentine, pour les raisons suivantes :

- le segment antérieur utriculaire de l'adulte procède du corps de la larve en forme de bursicule («bolsillo »);

- conformité de l'organe tribocytique de l'adulte avec celui de la métacercaire, qui est aussi elliptique et très allongé, avec sillon médian et entaille ventro-transversale très caractéristique (cf. Szidat, op. cit., fig. 2 et 3);

- présomption de Szidat (op. cit., p. 87) en faveur d'un petit mammifère carnivore ou encore d'un marsupial mangeur de batraciens comme hôte définitif;

- même localisation géographique (Argentine);

- même rapport dimensionnel des ventouses et absence d'œsophage.

Le genre Bursotrema se rapproche de Prudhoella Beverley-Burton, 1960, par la forme utriculaire du segment antérieur, la délimitation peu nette des deux segments 
du Ver, la forme allongée de l'organe tribocytique et la localisation des vitellogènes dans la partie moyenne du corps. Il s'en distingue par la présence d'un cône génital et par la situation du réservoir vitellin au niveau intertesticulaire.

Pour les deux genres, la forme allongée de l'organe tribocytique (comme chez Alaria Schrank) et l'adaptation aux Mammifères plaident en faveur de leur attribution à la sous-famille des Alariinae.

Didelphodiplostomum nunezae n. sp. (fig. 3).

Dans le même hôte et le même lieu, à la même date, la $\mathrm{D}^{\text {ra }} \mathrm{O}$. de Núñez a recueilli un matériel représentant une espèce congénérique du type $D$. variabile (Chandler, 1932) Dubois, 1944.

DiAGNOSE (avec les caractères du genre) (2). - Corps bisegmenté long de 1,05 à $1,33 \mathrm{~mm}$, à constriction transversale bien marquée. Segment antérieur cochléariforme, arrondi à l'état contracté, mesurant $0,57-0,64$ / 0,60-0,75 $\mathrm{mm}$, à extrémité céphalique munie de pseudo-ventouses cupuliformes; segment postérieur ovoïde à conique, subégal en longueur mais plus étroit, 0,50-063/0,45$0,61 \mathrm{~mm}$. Rapport des dimensions longitudinales du second au premier $=0,8-1$. Pharynx ellipsoïdal et fortement musculeux, 105$120 / 85-100 \mu$, toujours plus long que la ventouse buccale qui mesure 85-115/105-125 $\mu$; ventouse ventrale allongée transversalement, plus grande que cette dernière, 85-95/140175. Esophage court. Organe tribocytique très développé, arrondi ou ovale, 270-340/ 250-340 $\mu$, s'ouvrant par une large fente médiane, irrégulière, et recouvrant partiellement l'acetabulum.

Ovaire submédian à latéral, situé à la limite intersegmentaire ou tout au début du segment postérieur, 95-105/160-230 $\mu$. Testicules trilobés (le premier asymétriquement développé), à lobe médian dorsal, commissural, et à lobes latéraux divisés en deux lobules; testicule antérieur mesurant 400 à $500 \mu$ transversalement, 120 à $160 \mu$ et 210 à $240 \mu$ pour l'un et l'autre de ses lobes latéraux

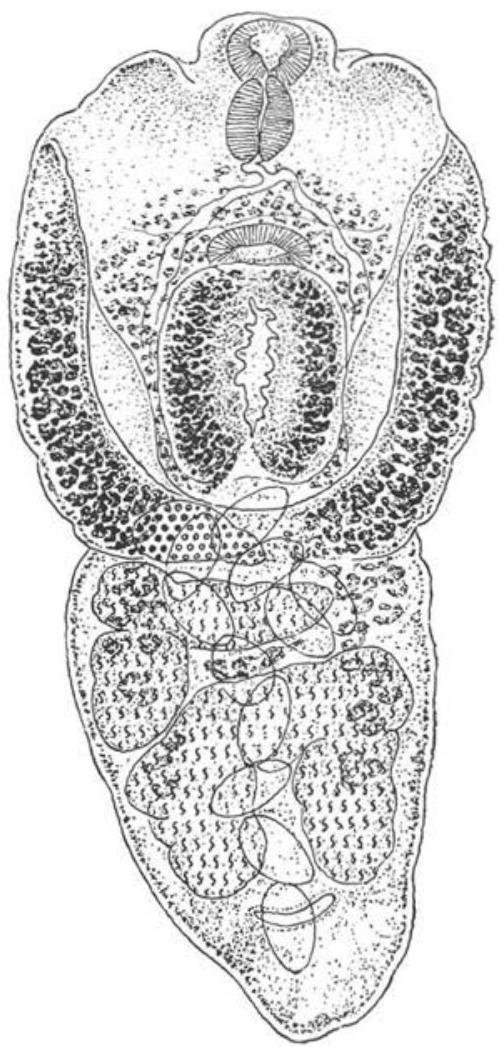

FIG. 3. - Didelphodiplostonum numezae n. sp., de Didelphis azarae Temm. Holotype. Longueur 1,24 mm. Vue ventrale.

(2) Cf. Synopsis des Strigeidae et des Diplostomatidae (Trematoda), p. 626. 
dans le sens longitudinal, 125-210/190-200 $\mu$ pour le lobe médian dorsal; testicule postérieur mesurant 330 à $490 \mu$ transversalement, 190 à $245 \mu$ et 250 à $290 \mu$ pour l'un et l'autre de ses lobes latéraux dans le sens longitudinal, 125-200/180$230 \mu$ pour le lobe médian dorsal. Vésicule séminale suive d'une petite poche éjaculatrice (visible sur les coupes). Vitellogènes à répartition plus étendue et à densité beaucoup plus forte dans le segment antérieur, où ils s'avancent jusqu'au niveau œsophagien et envahissent l'organe tribocytique; raréfiés dans le segment postérieur, où ils sont réduits à deux semis ventro-latéraux s'étendant jusqu'à la mi-longueur du second testicule. Réservoir vitellin intertesticulaire. Bourse copulatrice de dimensions réduites, à pore dorsal, subterminal. Absence de cône génital. Eufs très grands, 115-122/68$78 \mu$, à coque épaisse de 5 à $12 \mu$, et dont le nombre peut atteindre 14 .

HôTE: Didelphis azarae Temminck, 1825.

HABITAT : intestin.

Localıté : Castelar, prov. Buenos Aires (Argentine).

Matériel: Museo Argentino de Ciencas Naturales «Bernardino Rivadavia », av. Angel Gallardo 470, Buenos Aires, coll. helm. n 275/1-17 (O. de Núñez), 275/18 (holotype et 2 paratypes), 275/19-20 (4 2 paratypes), 275/21 (coupes histologiques); Université de Neuchâtel (G.D.), n ${ }^{\circ}$ W 19 et 20 (paratypes).

Discussion. - La nouvelle espèce, que nous dédions à la $\mathrm{D}^{\text {rn }}$ Ostrowski de Núñez, se distingue du type générique, Didelphodiplostomum variabile (Chandler, 1932) Dubois, 1944, par les caractères suivants :

- grosseur des œufs (115-122/68-78 $\mu$, au lieu de 75-100/50-65 $\mu$ ), à parois épaisses :

- rapport des longueurs du segment postérieur au segment antérieur $(0,8-1$, au lieu de $0,57-0,66)$;

- ventouse ventrale plus grande que la ventouse buccale (ces organes sont subégaux chez D. variabile);

- hôte limité à l'Amérique du Sud (République Argentine, Uruguay, Paraguay et Brésil oriental, jusque dans les états de Paraiba et de Ceara), tandis que celui du générotype (Didelphis marsupialis virginiana Kerr.) est propre à l'Amérique du Nord.

Le caractère «paralarien » de Didelphodiplostomum nunezae n. sp. est accusé par la rareté, la faible densité des follicules vitellogènes dans le segment postérieur, par le grand développement de l'organe tribocytique et par la trilobation des testicules [cf. Alaria (Paralaria) mustelae canadensis Webst. et Wolfg., A. (P.) pseudoclathrata (Krause) et $A$. (P.) taxideae Swans. et Ericks.]. Il est mis aussi en relief par la grosseur du pharynx [cf. $A$. (P.) taxideae]. Ainsi se confirme la position systématique du genre Didelphodiplostomum Dub. dans la sous-famille des Alariinae. 


\section{Bibliographie}

BFVFrLeY-BURTON (M.), 1960. - Some Trematodes from Otters in Southern Rhodesia including a new Strigeid, Prudhoella rhodesiensis, n. gen., n. sp. Proc. helminth. Soc. Wash., 27, 129-134.

CHANDler (A. C.), 1932. - Notes on the helminth parasites of the opossum (Didelphis virginiana) in Southeast Texas, with descriptions of four new species. Proc. U.S. Nat. Mus., 81, Art. 16, 1-15.

DuBois (G.), 1970. - Synopsis des Strigeidae et des Diplostomatidae (Trematoda). Mém. Soc. neuchâtel. Sci. nat., 10, 259-727.

Szidat (L.), 1960. - Nuevo tipo larvas de Alariinae (Trematoda) del riñon de Leptodactylus ocellatus de la Argentina. Neotropica, 6, 82-88. 